Pneumologe 2018 $\cdot 15: 396-403$ https://doi.org/10.1007/s10405-018-0195-5 Online publiziert: 26. Juni 2018

(c) Der/die Autor(en) 2018

\section{Redaktion}

M. Kreuter, Heidelberg

U. Müller-Ladner, Bad Nauheim

T. Wessendorf, Essen

CrossMark
H. Olschewski

Klinische Abteilung für Pulmonologie, Medizinische Universitätsklinik Graz, Graz, Österreich

\title{
Pulmonale Hypertonie und rheumatische Erkrankungen
}

der über einen pulmonal arteriellen Ballonkatheter bestimmt wird („pulmonary arterial wedge pressure“, PAWP), höchstens $15 \mathrm{~mm} \mathrm{Hg}$ beträgt, wird angenommen, dass die pulmonale Hypertonie nicht die Folge einer Herzkrankheit ist [3]. Wenn in der Lungenfunktionsuntersuchung die Vitalkapazität über $70 \%$ liegt und in der Computertomographie (CT) des Thorax weniger als $10 \%$ des Lungenvolumens eine interstitielle Veränderung aufweist, wird angenommen, dass die pulmonale Hypertonie nicht die Folge einer Lungenfibrose ist [4]. Wenn sich im Rahmen einer Kollagenose eine Lungenfibrose entwickelt, so liegt auch ein erhöhtes Risiko für eine pulmonale Hypertonie vor. In einer englischen Kohorte mit Patienten mit systemischer Sklerose (SSc) war die Kombination von pulmonaler Hypertonie und Lungenfibrose mit einer besonders schlechten 3-Jahres-Überlebensrate von nur $28 \%$ assoziiert [5]. In den zulassungsorientierten Studien für PAH-Medikamente wurden solche Patienten jedoch stets ausgeschlossen, weil es sich bei diesen Krankheiten nicht um eine ,isolierte pulmonale Hypertonie“ im Sinne einer PAH handelt. Die folgenden Ausführungen werden sich dann auch ausschließlich auf die rheumatischen Erkrankungen mit PAH beziehen, nachdem es zu den anderen Formen der pulmonalen Hypertonie kaum Register- oder Studiendaten gibt.

\section{Pathologie und Patho- physiologie}

Seit Langem ist bekannt, dass die kleinen Lungengefäße im Rahmen von Kollage- nosen ein Remodeling entwickeln können, welches der idiopathischen PAH (IPAH) histologisch und funktionell sehr stark ähnelt. Dieses Remodeling führt in weiterer Folge zu einem Anstieg des pulmonal arteriellen Drucks. Typischerweise erreichen die Patienten mit den Kollagenosen weniger hohe pulmonale Druckwerte, als die Patienten mit der IPAH. Das dürfte mehrere Gründe haben: Die Patienten mit Kollagenosen weisen durchschnittlich ein höheres Alter auf und im höheren Alter werden nicht mehr so hohe Druckwerte erreicht wie bei jüngeren Patienten [6]. Es gibt aber auch Evidenz dafür, dass die Kollagenosen zu einer Beeinträchtigung der kardialen Reserve führen [7]. Das äußert sich darin, dass bei gegebener Nachlast (erhöhter PAP) das Herzzeitvolumen stärker reduziert und die Vorlast (rechtsatrialer Druck) stärker erhöht ist. Es ist experimentell nicht gut belegt, aber zu erwarten, dass dann unter Belastung nur ein geringer Anstieg des Herzzeitvolumens möglich ist. Ein solcher Befund war in einem Kollektiv mit überwiegend IPAHPatienten der stärkste Prädiktor der Mortalität [8]. Dieser Zusammenhang würde auch erklären, warum die Kollagenosepatienten in allen Studien durch einen besonders stark reduzierten 6-MinutenGehtest auffallen.

Bei Menschen mit weißer Hautfarbe ist unter allen Kollagenosen die SSc am stärksten mit einer pulmonalen Hypertonie assoziiert. Im asiatischen Raum scheint dagegen der systemische Lupus erythematodes (SLE) die häufigste Ursache einer kollagenoseassoziierten $\mathrm{PAH} \mathrm{zu}$ sein [9]. Nicht selten findet man Mischkollagenosen unter den Pati- 
Hier steht eine Anzeige.

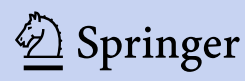


enten mit einer kollagenoseassoziierten PAH. Deutlich seltener sind es Patienten mit einer rheumatoiden Arthritis, einer Dermatomyositis, einem SjögrenSyndrom oder einem AntisynthetaseSyndrom. Unter solchen Patienten ist die PAH zwar selten, kann aber im Einzelfall besonders schwer sein und aggressiv verlaufen. Beim SLE wurde immer wieder über eine Besserung der pulmonalen Hämodynamik unter immunsuppressiver Therapie berichtet. Solche Erfahrungen wurden bei anderen Kollagenosen leider nicht gemacht.

\section{》) Der Pathomechanismus des pulmonal vaskulären Remodeling ist nicht endgültig aufgeklärt}

Bei keiner dieser Erkrankungen ist der Pathomechanismus des pulmonal vaskulären Remodeling endgültig aufgeklärt. Es ist $\mathrm{zu}$ vermuten, dass im Rahmen der Autoimmunität gefäßaktive Substanzen entstehen, die zur Konstriktion und zur Proliferation der pulmonalen Gefäßwände führen. In einer multizentrischen Untersuchung aus Deutschland wurden aktivierende Autoantikörper gegen den Endothelin-A-Rezeptor und den Angiotensin-1-Rezeptor nachgewiesen, die sicherlich zu Vasokonstriktion und Proliferation der Gefäßwand beitragen können [10]. Die Autoantikörper wurden in einer unabhängigen amerikanischen Kohorte bestätigt, obwohl die Autoren dieser Arbeit Zweifel an der Spezifität dieses Befundes für Kollagenosen haben [11].

Ein besonderes Merkmal von Kollagenosen ist die Anhäufung von Kollagen im Interstitium. Bei der SSc findet sich in den Lungengefäßen eine charakteristische Zunahme von Kollagen 14 und Kollagen 18. Endostatin ist das wichtigste Abbauprodukt von Kollagen 18 und kann auch in der Zirkulation nachgewiesen werden [12]. Endostatin hat starke angiostatische Eigenschaften, das heißt, es reduziert die Vaskularisierung des Gewebes und damit dessen Versorgung mit Sauerstoff und Nährstoffen. In zwei unabhängigen Kohorten wurden bei SSc mit $\mathrm{PAH}$ erhöhte Konzentrationen von En- dostatin im Blut nachgewiesen [12, 13]. Interessanterweise gibt es einen genetischen Polymorphismus, der die Bildung von Endostatin beeinflusst. Bereits ein Genotyp mit erhöhter Endostatinbildung ist mit einer deutlich schlechteren Prognose bei SSc-assoziierter PAH verbunden [13]. Die zirkulierenden Endostatinspiegel sind stärker mit der kardialen Funktion als mit dem pulmonal vaskulären Druck korreliert [12]. Das spricht dafür, dass das Endostatin eine besonders wichtige Rolle für das rechtsventrikuläre Versagen hat. Das dürfte durch seine angiostatischen Eigenschaften zu erklären sein, welche möglicherweise kritisch für die Perfusion des Myokards sind.

Bei der SSc werden zwei Subgruppen unterschieden: die diffuse systemische Sklerose und die sog. limitierte SSc. Bei Letzterer weisen Patienten meistens AntiZentromer-Antikörper auf, während die Patienten mit der diffusen SSc überwiegend Scl-70-Antikörper zeigen und nur selten eine isolierte schwere PAH entwickeln. Ein Teil der Patienten mit limitierter SSc wurde früher als CREST-Syndrom bezeichnet (CREST: Calcinosis cutis, Raynaud-Syndrom, Ösophagusbeteiligung, Sklerodaktylie, Teleangiektasie). Bei einem Teil dieser Patienten kommt es zu einer ,isolierten“ PAH. Der Begriff „isoliert“ wird verwendet, um zu verdeutlichen, dass die Dünnschicht-Computertomographie (CT) in solchen Fällen keine eindeutigen Zeichen einer Lungenfibrose zeigt und dass keinerlei Hinweise auf eine Linksherzerkrankung vorliegen. Obwohl viele Patienten mit limitierter SSc nie eine PAH entwickeln, stellte diese Subgruppe die Mehrheit der Patienten mit kollagenoseassoziierter PAH dar, die an den kontrollierten randomisierten Studien für die gezielten PAH-Medikamente teilgenommen und damit zur Zulassung in dieser Indikation beigetragen haben.

\section{Epidemiologie}

Wenn große Kohorten von SSc-Patienten systematisch auf eine pulmonale Hypertonie untersucht wurden, so fanden sich 5-12\% mit einer manifesten PAH. Studien mit höheren Prävalenzzahlen sind mit größter Vorsicht zu interpretieren, weil entweder keine Rechtsherzkatheterkontrolle erfolgte oder die Lungenfunktionsund CT-Kriterien für den Ausschluss einer Non-PAH PH nicht rigoros überprüft wurden.

Für die SSc-assoziierte PAH, aber auch für alle anderen kollagenoseassoziierten PAH-Fälle findet sich ein Geschlechtsverhältnis weiblich zu männlich von 4:1 bis 8:1. Das mittlere Alter bei Diagnose ist häufig über 60 Jahre. Die Überlebenszeit ist deutlich kürzer als bei vergleichbar hämodynamisch betroffenen Patienten mit einer IPAH. Tatsächlich ist von einer knapp 3-fach höheren Mortalität auszugehen als bei IPAH. Prinzipiell ist die pulmonale Symptomatik sehr ähnlich wie bei den Patienten mit der IPAH. Bei Diagnose sind allerdings die pulmonal arteriellen Druckwerte deutlich niedriger als bei Patienten mit einer IPAH. Das mag neben den oben angesprochenen pathophysiologischen Unterschieden damit zusammenhängen, dass Kollagenosepatienten sich wegen ihrer Kollagenose häufig bereits in fachärztlicher Betreuung befinden und daher der PAH eine erhöhte Aufmerksamkeit zu Teil wird.

\section{Diagnostik}

Im Rahmen der Diagnostik spielt die Dünnschicht-CT eine besondere Rolle, weil bei Kollagenosen einerseits mit interstitiellen Lungenveränderungen gerechnet werden muss und weil die $\mathrm{Pa}$ tienten nicht nur an den pulmonalen Arterien, sondern auch an den pulmonalen Venen von einem obstruktiven Remodeling betroffen sein können. Sie entwickeln dann das klinische Bild einer pulmonal venookklusiven Erkrankung (PVOD). Die PVOD lässt sich im CT an verdickten interlobulären Septen und multiplen zentrilobulären Ödeminseln erkennen. Zusätzlich ist sie nicht selten mit Pleuraergüssen und verdickten mediastinalen Lymphknoten assoziiert. Nach der aktuellen Klassifikation zählen diese Fälle zur Gruppe 1'4.1 „Pulmonary veno-occlusive disease and/or pulmonary hemangiomatosis; associated with: connective tissue disease“.

Die Entwicklung einer isolierten $\mathrm{PAH}$ bei SSc ist mit einem Abfall der Diffusionskapazität für Kohlenmonoxid 
Hier steht eine Anzeige.

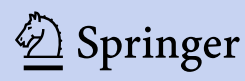


(DLCO) assoziiert [14]. Deswegen gilt eine erniedrigte DLCO unter $60 \%$ der Norm als ein Indikator einer möglichen PAH. Das gleiche gilt für einen Anstieg des BNP („brain natriuretic peptide“), welches bei der kollagenoseassoziierten $\mathrm{PAH}$ praktisch immer erhöht ist. Allerdings ist dieser Marker unspezifisch und steigt auch bei jeder Art von linksventrikulärer Funktionsstörung an. Die DETECT-Studie hat bei SSc-Patienten mit einer $\mathrm{DLCO}<60 \%$ systematisch nach Faktoren gesucht, welche mit einer manifesten PAH assoziiert waren. Dabei zeigte die Echokardiographie per se zwar eine enttäuschende Aussagekraft im Grenzwertbereich des PAP um die $25 \mathrm{~mm} \mathrm{Hg}$ herum, jedoch konnte in $\mathrm{Zu}$ sammenschau mit den anderen Markern ein sinnvoller Risikoscore für eine $\mathrm{PAH}$ bei SSc abgeleitet werden [15]. Generell gilt, dass eine Rechtsherzkatheteruntersuchung vor der Einleitung einer PAHTherapie obligatorisch gefordert wird, allein um die PAH nachzuweisen (Druckund PVR-Kriterium) und um eine begleitende Linksherzerkrankung sicher auszuschließen.

\section{》) Eine erniedrigte DLCO unter $60 \%$ der Norm gilt als PAH- Indikator}

Bei allen Kollagenosepatienten mit Luftnot bei Belastung oder anderen möglichen Symptomen einer PAH wird von den Leitlinien eine echokardiographische Abklärung empfohlen. Bei asymptomatischen Patienten gilt diese Empfehlung allerdings nur für die SSc. Bei diesen Patienten wird eine jährliche echokardiographische Kontrolle ausdrücklich empfohlen, weil in einem Intervall von durchschnittlich 7 Jahren in knapp 15\% der Fälle mit der Manifestierung einer PAH $\mathrm{zu}$ rechnen ist. Liegt der PAP bereits bei der ersten Untersuchung zwischen 21 und $24 \mathrm{~mm} \mathrm{Hg}$, ist dieses Risiko dafür allerdings fast 4 -fach höher [16]. In unserer eigenen prospektiven Studie an einer Kohorte von SSc-Patienten, war über einen Beobachtungszeitraum von 4 Jahren kein signifikanter Anstieg des PAP in Ruhe festzustellen, wohl aber ein Anstieg des PVR, ein Anstieg des pulmonalen

Pneumologe 2018 $\cdot 15: 396-403$ https://doi.org/10.1007/s10405-018-0195-5

(c) Der/die Autor(en) 2018

\section{H. Olschewski}

\section{Pulmonale Hypertonie und rheumatische Erkrankungen}

\section{Zusammenfassung}

Nach der idiopathischen pulmonal arteriellen Hypertonie (IPAH) stellen die mit einer Kollagenose (connective tissue disease [CTD]) assoziierten pulmonal arteriellen Hypertonien (PAH) die zweitgrößte Subgruppe innerhalb der PAH-Gruppe dar. Am häufigsten betroffen von einer PAH sind die Sklerodermie und Mischkollagenosen. Aber auch der systemische Lupus erythematodes (SLE), die Dermatomyositis, das Sjögren-Syndrom, Antisynthetase-Syndrom und die rheumatoide Arthritis weisen ein erhöhtes PAH-Risiko auf. Die pulmonale Symptomatik und die Therapie sind ähnlich wie bei der IPAH. Diagnostisch wird für die systemische Sklerose (SSc) ein jährliches echokardiographisches Screening auf eine pulmonale Hypertonie empfohlen. Bei den anderen Kollagenosen wird eine solche Diagnostik dann empfohlen, wenn Symptome vorliegen, die zu einer pulmonalen Hypertonie passen, insbesondere Luftnot bei Belastung, Müdigkeit oder Schwächeanfälle. Eine frühzeitige Therapie mit PAHMedikamenten wird empfohlen, nachdem die Prognose einer CTD assoziierten PAH deutlich ungünstiger ist, als bei einer IPAH. Auf eine kollagenoseassoziierte pulmonal venookklusive Erkrankung (PVOD) als Differenzialdiagnose ist besonders zu achten, weil sie ein hohes Risiko für therapieassoziierte Nebenwirkungen mit sich bringt. Zugelassen für die kollagenoseassoziierte $\mathrm{PAH}$ sind Endothelinrezeptorantagonisten (ERA), Phosphodiesterase-5-Inhibitoren (PDE5i), IPRezeptoragonisten (IPA) und ein Stimulator der löslichen Guanylatzyklase (sGCS). In der Kombination mit immunsuppressiven Substanzen und wegen der Besonderheiten der Grundkrankheit ist unter PAH-Medikamenten mit mehr und schwereren Nebenwirkungen zu rechnen als bei IPAH-Patienten.

\section{Schlüsselwörter}

Kollagenosen · Bindegewebserkrankungen - Idiopathische pulmonal arterielle Hypertonie . Pulmonale venookklusive Erkrankung . Diagnosetechniken

\section{Pulmonary hypertension and rheumatic diseases}

\section{Abstract}

After idiopathic pulmonary arterial hypertension (IPAH), connective tissue disease (CTD)-associated PAH represents the largest subgroup within the PAH group of diseases. Systemic sclerosis ( $\mathrm{SSC}$ ) and mixed connective tissue diseases are at the highest risk of PAH; however, systemic lupus erythematosus (SLE), dermatomyositis, Sjögren's syndrome, antisynthetase syndrome and rheumatoid arthritis also show an increased risk for PAH. The pulmonary symptoms and treatment are similar to those for IPAH. For SSC a yearly echocardiographic screening for PAH is recommended. In the other CTDs this diagnostic measure is recommended only if signs and symptoms of PAH occur, particularly dyspnea on exertion, fatigue or fainting/syncope. An early therapy with targeted PAH medication is recommended, as the prognosis of CTD-associated PAH is much worse compared to IPAH. A CTD- associated pulmonary veno-occlusive disease (PVOD) must be considered as a differential diagnosis, because this disease is particularly prone to treatment-associated side effects. Approved for targeted collagen-associated PAH are endothelin receptor antagonists (ERA), phosphodiesterase 5 inhibitors (PDE5i), IP receptor agonists (IPA) and stimulators of soluble guanylate cyclase (sGCS). When combined with immunosuppressants and because of the special properties of the underlying diseases, targeted PAH medications are associated with higher risks of severe side effects compared to IPAH patients.

\section{Keywords}

Collagen diseases - Connective tissue diseases - Idiopathic pulmonary arterial hypertension - Pulmonary veno-occlusive disease $\cdot$ Diagnostic techniques 
Hier steht eine Anzeige.

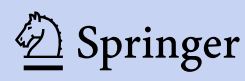


Drucks bei einer Belastung mit $50 \mathrm{~W}$ auf dem Ergometer und ein Abfall der höchsten erreichten Sauerstoffaufnahme (peak $\mathrm{VO}_{2}$ ). Bei $3 \%$ der Patienten wurde eine PAH manifest [17]. Das bedeutet, dass der pulmonale Druck in Ruhe nicht wirklich sensitiv für das subtile Fortschreiten des pulmonal vaskulären Remodeling bei SSc ist, die Ergometrie und insbesondere die Hämodynamik unter Belastung aber eine Verbesserung der Sensitivität ermöglicht.

\section{Therapie}

Die Therapie der PAH bei rheumatologischen Krankheiten folgt den gleichen Prinzipien wie die Therapie der IPAH. Allerdings sind meist die Therapieeffekte weniger eindrucksvoll und die Nebenwirkungen sind dafür schwerer. Viele Patienten mit rheumatologischen Erkrankungen erhalten heutzutage immunsuppressive Therapien oder Biologika, obwohl keines dieser Medikamente durch Leitlinien in der Langzeittherapie abgesichert ist [18]. Der dennoch häufige Einsatz kann zu Interaktionen mit den PAHMedikamenten führen und die Komplikationen solcher Medikamente können die Nebenwirkungen und Komplikationen von PAH-Medikamenten verstärken. Dies ist besonders relevant, wenn immunsuppressive Therapien zu akuten septischen Komplikationen führen, die einen Vasomotorenkollaps mit systemischer Hypotonie zur Folge haben. Diese Komplikation kann unter der Einwirkung von PAH-Medikamenten, die bekanntlich alle starke Vasodilatoren sind, erheblich aggraviert werden.

Anders als bei der IPAH findet man sehr selten Patienten, die zur Gruppe der „Kalziumantagonisten-Responder" gehören. Wird dennoch anhand der akuten hämodynamischen Kriterien bei der Rechtsherzkatheteruntersuchung ein solcher Responder identifiziert, muss man damit rechnen, dass trotz regelmäßiger Einnahme des Kalziumantagonisten die pulmonale Hypertonie nach wenigen Monaten oder Jahren fortschreitet und dass man alternativ oder zusätzlich andere gezielte PAH-Medikamente einsetzen muss.

Bei Kollagenosepatienten mit PAH wurden unter Monotherapien mit Endo- thelinrezeptorantagonisten (ERA), Phosphodiesterase-5-Inhibitoren (PDE5i), Prostanoiden oder Selexipag, einem nichtprostanoidischen IP-Rezeptorantagonisten (IPA) und einem Stimulator der löslichen Guanylatzyklase (sGCS) eindeutige Therapieerfolge dokumentiert. Die eindrucksvollsten Therapieergebnisse ließen sich allerdings unter der initialen Kombinationstherapie mit dem ERA Ambrisentan und dem PDE5i Tadalafil darstellen [19]. Tatsächlich werden viele Patienten relativ frühzeitig auf eine Kombinationstherapie mit einem modernen ERA und einem PDE5i eingestellt. Diese Therapien sind in aller Regel gut verträglich, dennoch muss mit Kopfschmerzen, Flush-Syndrom, Beinödemen, symptomatischer Hypotonie und einer chronischen Anämie gerechnet werden. Unter den modernen Medikamenten ist die Hepatotoxizität eine absolute Rarität.

Bosentan, ein ERA der ersten Generation, weist umfangreiche Arzneimittelinteraktionen auf. Das betrifft beispielsweise Vitamin-K-Antagonisten und Kontrazeptiva sowie andere PAH-Medikamente. Die Kombination von Bosentan und Cyclosporin A ist regelrecht kontraindiziert. Bosentan ist mit einer kumulativen 1-Jahres-Hepatotoxizität von ca. $10 \%$ belastet [20]. Das macht auch im Langzeitverlauf monatliche Kontrollen der Transaminasen erforderlich. Bis heute stellt aber Bosentan das einzige für akrale $\mathrm{Ne}$ krosen bei SSc (früher: Rattenbissnekrosen) zugelassene Medikament dar und wird daher noch relativ häufig verordnet, insbesondere, wenn die hämodynamischen Kriterien für eine $\mathrm{PAH}$ nicht erfüllt sind.

Anders als bei Patienten mit IPAH treten unter PAH-Medikamenten vermehrt Hypoxämien auf, insbesondere wenn bereits vor der Therapie Gasaustauschstörungen erkennbar waren. Dies dürfte mit einer gestörten Gefäßarchitektur in der Lunge zu tun haben, die dadurch charakterisiert ist, dass nicht nur verengte, sondern auch deutlich dilatierte mikrovaskuläre Pulmonalgefäße vorkommen. Dieses Problem kann die Optionen für PAH-Medikamente im Einzelfall erheblich einschränken. Mischkollagenosen sind von diesem klinischen Prob- lem besonders häufig betroffen. In jedem einzelnen Fall sollte vor Therapiebeginn eine Dünnschicht-CT der Lunge erfolgen. Bei zunehmender Hypoxämie unter $\mathrm{PAH}-$ Therapie sollte diese erneut durchgeführt und sorgfältig auf Zeichen einer PVOD geprüft werden, weil das keine ganz seltene pulmonal vaskuläre Manifestation von Kollagenosen darstellt.

》) Vor der Therapie sollte stets
eine Rechtsherzkatheteruntersu-
chung und eine Dünnschicht-CT
der Lunge erfolgen

Wenn bei einer rheumatologischen Grunderkrankung eine PAH fortschreitet und sich trotz bestmöglicher PAHTherapie nicht stabilisieren lässt, so kommt mitunter eine Lungentransplantation in Frage. Häufig ist allerdings die Altersgrenze bereits überschritten oder es liegen Komorbiditäten vor, die diesen Eingriff verbieten, wobei die Refluxösophagitis bei der SSc hierbei keine Kontraindikation darstellt. Obwohl die Transplantation bei solchen Patienten mit einem höheren Risiko verbunden ist als bei der IPAH, gilt eine rheumatologische Grundkrankheit nicht als Kontraindikation zur Lungentransplantation [1]. Als Überbrückung zur Lungentransplantation oder als palliative Therapiemaßnahme bei ausgereizter medikamentöser Therapie kommt eine atriale Septostomie in Frage. Dieser Eingriff verbessert sehr rasch die Füllung des linken Ventrikels und entlastet den rechten Ventrikel, allerdings führt er auch sofort zu einer deutlichen Verschlechterung der Hypoxämie [21]. Dieser Eingriff bleibt erfahrenen Zentren für pulmonale Hypertonie vorbehalten.

\section{Fazit für die Praxis}

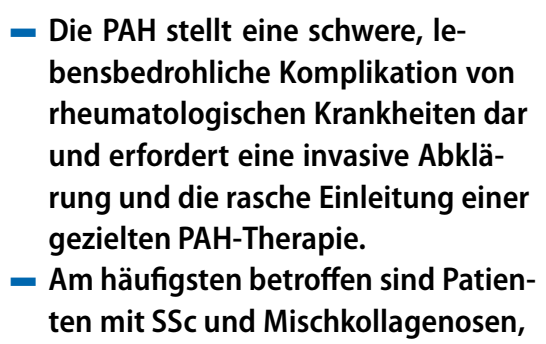


aber andere Kollagenosen gehen ebenfalls mit einem erhöhten PAHRisiko einher.

- Für die SSc wird ein jährliches echokardiographisches Screening auf PAH empfohlen, für die anderen Kollagenosen nur bei PAH-Symptomen und -Zeichen.

- Bei der PAH ist ein interdisziplinär abgestimmtes Vorgehen von größter Bedeutung, weil mit mehr Nebenwirkungen und Therapieinteraktionen zu rechnen ist als bei Patienten mit IPAH.

- Besonders zu beachten ist die kollagenoseassoziierte PVOD, die ein besonderes Risiko für Nebenwirkungen und Komplikationen unter PAHMedikamenten darstellt.

\section{Korrespondenzadresse}

\section{Prof. Dr. H. Olschewski}

Klinische Abteilung für Pulmonologie,

Medizinische Universitätsklinik Graz

Auenbruggerplatz 20, $8036 \mathrm{Graz}$, Österreich horst.olschewski@medunigraz.at

Funding. Open access funding provided by Medical University of Graz.

\section{Einhaltung ethischer Richtlinien}

Interessenkonflikt. H. Olschewski ist als Berater, Redner und Mitglied in verschiedenen Boards tätig für Actelion, Bayer, Bellerophon, MSD, Pfizer, Novartis, Boehringer, Roche, und Inventiva.

Dieser Beitrag beinhaltet keine von den Autoren durchgeführten Studien an Menschen oder Tieren.

Open Access. Dieser Artikel wird unter der Creative Commons Namensnennung 4.0 International Lizenz (http://creativecommons.org/licenses/by/4.0/deed. de) veröffentlicht, welche die Nutzung, Vervielfältigung, Bearbeitung, Verbreitung und Wiedergabe in jeglichem Medium und Format erlaubt, sofern Sie den/die ursprünglichen Autor(en) und die Quelle ordnungsgemäßnennen, einen Linkzur Creative Commons Lizenz beifügen und angeben, ob Änderungen vorgenommen wurden

\section{Literatur}

1. Galie N, Humbert M, Vachiery JL et al (2015) Cardiology 2015(46):903-975

2. Kovacs G, Berghold A, Scheidl S, Olschewski H (2009) Pulmonary arterial pressure during rest and exercise in healthy subjects: a systematic review. Eur Respir J 34:888-894
3. Vachiery JL, Adir Y, Barbera JA et al (2013) Pulmonary hypertension due to left heart diseases. J Am Coll Cardiol 62:D100-D108

4. Seeger W, Adir Y, Barbera JA et al (2013) Pulmonary hypertension in chronic lung diseases. J Am Coll Cardiol 62:D109-D116

5. Condliffe R, Kiely DG, Peacock AJ et al (2009) Connective tissue disease-associated pulmonary arterial hypertension in the modern treatment era. Am J Respir Crit Care Med 179:151-157

6. Hoeper MM, Huscher D, Ghofrani HA et al (2013) Elderly patients diagnosed with idiopathic pulmonary arterial hypertension: results from the COMPERA registry. Int J Cardiol 168:871-880

7. Hsu S, Houston BA, Tampakakis E et al (2016) Right ventricular functional reserve in pulmonary arterial hypertension. Circulation 133:2413-2422

8. Chaouat A, Sitbon O, Mercy Met al (2014) Prognostic value of exercise pulmonary haemodynamics in pulmonary arterial hypertension. Eur Respir J 44:704-713

9. Hao YJ, Jiang X, Zhou W et al (2014) Connective tissue disease-associated pulmonary arterial hypertension in Chinese patients. Eur Respir J 44(4):963

10. Becker MO, Kill A, Kutsche M et al (2014) Vascular receptor autoantibodies in pulmonary arterial hypertension associated with systemic sclerosis. Am J Respir Crit Care Med 190:808-817

11. Bourji Kl, Khair RM, Damico RL et al (2015) Vascular receptor autoantibodies in pulmonary arterial hypertension. Am J Respir Crit Care Med 191:602-2267LE

12. Hoffmann J, Marsh LM, Pieper M et al (2015) Compartment-specific expression of collagens and their processing enzymes in intrapulmonary arteries of IPAH patients. Am J Physiol Lung Cell Mol Physiol 308:L1002-L1013

13. Damico R, Kolb TM, Valera L et al (2015) Serum endostatin is a genetically determined predictor of survival in pulmonary arterial hypertension. Am J Respir Crit Care Med 191:208-218

14. Steen V, Medsger TA Jr. (2003) Predictors of isolated pulmonary hypertension in patients with systemic sclerosis and limited cutaneous involvement. Arthritis Rheum 48:516-522

15. Coghlan JG, Denton CP, Grunig E et al (2014) Evidence-based detection of pulmonary arterial hypertension in systemic sclerosis: the DETECT study. Ann Rheum Dis 73:1340-1349

16. Valerio CJ, Schreiber BE, Handler CE, Denton CP, Coghlan JG (2013) Borderline mean pulmonary artery pressure in patients with systemic sclerosis: transpulmonary gradient predicts risk of developing pulmonary hypertension. Arthritis Rheum 65:1074-1084

17. Kovacs G, Avian A, Wutte N et al (2017) Changes in pulmonary exercise haemodynamics in scleroderma:a4-year prospective study. Eur Respir J.https:// doi.org/10.1183/13993003.01708-2016

18. Kowal-Bielecka O, Fransen J, Avouac J et al (2017) Update of EULAR recommendations for the treatment of systemic sclerosis. Ann Rheum Dis 76:1327-1339

19. Hassoun PM, Zamanian RT, Damico R et al (2015) Ambrisentan and Tadalafil up-front combination therapy in Scleroderma-associated pulmonary arterial hypertension. Am J Respir Crit Care Med 192:1102-1110

20. Humbert M, Segal ES, Kiely DG et al (2007) Results of European post-marketing surveillance of bosentan in pulmonary hypertension. Eur Respir J30:338-344
21. Reichenberger F, Pepke-Zaba J, McNeil K, Parameshwar J, Shapiro LM (2003) Atrial septostomy in the treatment of severe pulmonary arterial hypertension. Thorax 58:797-800 